\title{
Cancer immunotherapy: exploiting neoepitopes
}

\author{
Cell Research (2015) 25:887-888. doi:10.1038/cr.2015.66; published online 2 June 2015
}

The efficacy of cancer vaccines has long been hampered by insufficient definition of tumor-specific antigens. A recent study by Kreiter $e t$ al. published in Nature has provided a blueprint for a patient-tailored approach to develop individualized RNA vaccines.

For the past two decades, the concept of vaccination against cancer has suffered from insufficient definition of relevant target antigens. Consequently, clinical vaccination trials, typically targeting tumor-associated self-antigens, have generally failed to elicit therapeutic immunity in spite of detection of vaccine-induced T-cell responses in blood. In hindsight, these failures can be readily explained by the finding that many of these self-antigens are expressed in the thymus, resulting in deletion of the highly reactive T-cell repertoire and development of suppressive T-regulatory cells [1]. Moreover, circumvention of thymic tolerance by infusion of genetically engineered $\mathrm{T}$ cells targeting such antigens was found to be associated with severe toxicity in vital somatic tissues, vividly illustrating the physiological importance of immunological tolerance to many tumor-associated antigens [2].

In an almost ironic twist of science, clinical trials employing the two main 'competing' approaches for cancer immunotherapy, have shown us how to move forward with cancer vaccines. First, in-depth evaluation of the T-cell response in melanoma patients who responded to TIL-therapy, and infusion of ex vivo expanded tumor-infiltrating $\mathrm{T}$ cells, revealed that the T-cell clones driving therapeutic efficacy were not directed against lineage-specific and cancer testis antigens, but instead against neo-epitopes encoded by the tumor mutanome [3]. Essentially the same observation was subsequently made in patients responding to so-called checkpoint inhibitors, antibodies against cytotoxic T-lymphocyte-associated Protein 4 (CTLA-4) or the programmed cell death (PD)-1/PD-L1 axis, that can activate $T$ cells by neutralizing inhibitory pathways in T cells [4].

Since checkpoint inhibitors are not uniformly effective in every patient and every cancer type, while durable clinical responses frequently occur in the absence of overt manifestations of autoimmunity, it was fair to postulate that $T$ cells unleashed by checkpoint inhibitors recognize patient- and cancer-specific neoantigens derived from non-synonymous mutations rather than conserved self-antigens. Indeed, whole-exome sequencing of pre- and post-treatment tumor tissue has since revealed a strong association of the clinical response to checkpoint inhibition with the frequency of pre-treatment non-synonymous mutations in mouse models [5] and in human melanoma [4] and non-small cell lung cancer [6], two types of cancer with a particularly high mutational load.

These findings have set the stage for coordinated programs to identify patient-specific mutated antigens and target these through specific vaccines or transfer of antigen-specific $\mathrm{T}$ cells. Set aside the economic and regulatory challenges resulting from this highly individualized approach, several important questions have to be clarified: How can we predict which of the 50-500 mutations in a given tumor are immunogenic? How can we identify not only HLA class I-restricted epitopes, but also HLA class II-restricted CD4+ T-helper epitopes that are of critical importance to support the immune attack by CD8+ T cells and innate effector cells? How do we implement this information towards the design of patient-tailored vaccines?

The recent study by Kreiter and coworkers provided a blueprint for such an approach in the context of syngeneic mouse tumor models [7]. After identification of non-synonymous mutations through RNA and DNA sequencing and prioritization of the mutations based on expression level and prediction of presentation on MHC, animals were vaccinated using synthetic RNA vaccines encoding long peptides. Interestingly, the majority of immunogenic epitopes where found to be MHC class II-restricted [7]. The fact that non-synonymous cancer mutations are preferentially recognized by $\mathrm{CD} 4+\mathrm{T}$ cells may not be so surprising as the requirements for peptide processing and binding to MHC II are less restrictive than that for MHC I [8]. A downside of this flexibility is that prediction algorithms for MHC class II presentation suffers from inaccuracy compared to class I algorithms. Hence testing in pre-clinical models such as MHC-transgenic mice may be necessary to experimentally identify such epitopes, as exemplified by a recent study characterizing a class II neo-epitope of the glioma driver mutation IDH1R132H [9]. Complementary assays such as in situ proximity ligation assay [10] may aid the selection of neoepitopes presented on class II molecules by MHC class II + tumor cells or tumor- 
infiltrating antigen-presenting cells.

The efficacy of neoantigen-specific CD4+ T cells in controlling established tumors raises the question as to what their mechanism of action is. While direct cytotoxicity by CD4+ T cells [11] may play a role in tumors expressing $\mathrm{MHC}$ class II, more prominent effector mechanism probably include the orchestration of CD8 $+\mathrm{T}$ cells and innate effector cells, as well as the anti-tumor impact of cytokines such as interferon- $\gamma$ or tumor necrosis factor [12]. The study by Kreiter et al. suggests that a multimer vaccine targeting CD4 neoepitopes may act by unmasking CD8 epitopes previously not visible or not sufficiently visible to the immune system. The delineation of this complex process of antigen spreading to CD8 epitopes will be important in the future to help further tailor individualized (neo)antigen-specific cancer vaccines.

\section{Michael Platten ${ }^{1,2}$, Rienk Offringa $a^{3,4}$}

${ }^{1}$ DKTK Clinical Cooperation Unit Neuroimmunology and Brain Tumor Immunology, German Cancer Research Center, ${ }^{2}$ Neurology Clinic, University Hospital Heidelberg and National Center for Tumor Diseases, ${ }^{3}$ Dept. Molecular Oncology of Gastrointestinal Tumors, German Cancer Research Center, ${ }^{4}$ European Pancreas Center, Surgery Clinic, University Hospital Heidelberg, Heidelberg, Germany

Correspondence: Michael Platten ${ }^{\mathrm{a}}$,

Rienk Offringa ${ }^{\mathrm{b}}$

${ }^{a} E-m a i l: m . p l a t t e n @$ dkfz.de

bE-mail: r.offringa@dkfz.de

\section{References}

1 Klein L, Kyewski B, Allen PM, et al. Nat
Rev Immunol 2014; 14:377-391.

2 Morgan RA, Chinnasamy N, Abate-Daga D, et al. J Immunother 2013; 36:133-151.

3 Robbins PF, Lu YC, El-Gamil M, et al. Nat Med 2013; 19:747-752.

4 Snyder A, Makarov V, Merghoub T, et al. New Engl J Med 2014; 371:2189-2199.

5 Gubin MM, Zhang X, Schuster H, et al. Nature 2014; 515:577-581.

6 Rizvi NA, Hellmann MD, Snyder A, et al. Science 2015; 348:124-128.

7 Kreiter S, Vormehr M, van de Roemer N, et al. Nature 2015; 520:692-696.

8 Roche PA, Furuta K. Nat Rev Immunol 2015; 15:203-216.

9 Schumacher T, Bunse L, Pusch S, et al. Nature 2014; 512:324-327.

10 Bunse L, Schumacher T, Sahm F, et al. J Clin Invest 2015; 125:593-606.

11 Quezada SA, Simpson TR, Peggs KS, et al. $J$ Exp Med 2010; 207:637-650.

12 Braumuller H, Wieder T, Brenner E, et al. Nature 2013; 494:361-365. 